\title{
Dynamic Average Consensus and Consensusability of General Linear Multiagent Systems with Random Packet Dropout
}

\author{
Wen-Min Zhou ${ }^{1,2}$ and Jiang-Wen Xiao ${ }^{1,2}$ \\ ${ }^{1}$ School of Automation, Huazhong University of Science and Technology, Wuhan 430074, China \\ ${ }^{2}$ Key Laboratory of Image Processing and Intelligent Control of Education Ministry of China, Wuhan 430074, China \\ Correspondence should be addressed to Jiang-Wen Xiao; jwxiao@mail.hust.edu.cn
}

Received 10 June 2013; Revised 19 August 2013; Accepted 27 August 2013

Academic Editor: Jianquan Lu

Copyright (c) 2013 W.-M. Zhou and J.-W. Xiao. This is an open access article distributed under the Creative Commons Attribution License, which permits unrestricted use, distribution, and reproduction in any medium, provided the original work is properly cited.

\begin{abstract}
This paper is concerned with the consensus problem of general linear discrete-time multiagent systems (MASs) with random packet dropout that happens during information exchange between agents. The packet dropout phenomenon is characterized as being a Bernoulli random process. A distributed consensus protocol with weighted graph is proposed to address the packet dropout phenomenon. Through introducing a new disagreement vector, a new framework is established to solve the consensus problem. Based on the control theory, the perturbation argument, and the matrix theory, the necessary and sufficient condition for MASs to reach mean-square consensus is derived in terms of stability of an array of low-dimensional matrices. Moreover, mean-square consensusable conditions with regard to network topology and agent dynamic structure are also provided. Finally, the effectiveness of the theoretical results is demonstrated through an illustrative example.
\end{abstract}

\section{Introduction}

Multiagent systems (MASs) are kinds of networked systems in which each agent updates its states based on the information exchanges over communication networks. Due to their broad applications in many areas such as sensor networks [1], distributed computation [2], swarms and flocks [3], and formation control [4], the consensus problem of MASs has attracted increasing attention in recent years, and considerable interesting results have been obtained on this problem.

At the beginning, the consensus problem is investigated mainly for first-order and second-order MASs. Olfati-Saber and Murray [5] studied the consensus problem for a network of first-order integrators under various topology conditions, including directed or undirected, fixed or switching, and with or without communication time-delays; Hatano and Mesbahi [6] studied the asymptotic agreement of first-order continuous MASs over random information network based on stochastic stability analysis; Zhang and Tian [7] investigated the MASs consisting of discrete-time second-order agents under stochastic switching topology and proved that MASs can achieve mean-square consensus if and only if the union graphs in the topology set are connected. Sun et al. [8] investigated the finite-time consensus for first- and second-order leader-following Multiagent systems. In [9], Lin et al. further studied the consensus problems of a class of high-order Multiagent systems with dynamically changing topologies and time-delays and proved that the communication timedelays do not affect the stability of the Multiagent systems.

Recently, some researches on MASs with general linear dynamics have been conducted. Li et al. [10] proved that MASs with a communication topology having a spanning tree can reach consensus under an observe-type protocol if and only if each agent is stabilizable and detectable. $\mathrm{Xu}$ et al. [11] investigated the information structure for the consensusability of MASs under both fixed and switching topologies with dynamic output feedback control. In [12], Xu et al. further studied the leader-following consensus problem of discrete-time Multiagent systems with switching and 
undirected topology by applying two kinds of distributed observer-based consensus protocols to each agent. Su and Huang [13] proved that if the system matrix is marginally stable and the dynamic graph is jointly connected, both the leaderless and the leader-following consensus problems for linear discrete-time Multiagent systems under switching network topology can be solved.

Note that most of the existing results on the consensus problem of MASs are derived from the assumption that the network communication environment is perfect. However, communication constraints which may deteriorate the performance of MASs are inevitable in practice. There are numerous studies focusing on the consensus problem for MASs with either communication time delay or presence of perturbation or both of them, such as [14-18], but as one of the main reasons for performance deterioration, packet dropout, which takes place frequently during the information exchange between agents, is not fully investigated. Therefore, it is of great significance to study the effect of packet dropout in MASs. Ren and Beard [19] studied the consensus problem for first-order continuous-time agents communicating via unreliable network through dynamically changing interaction topology method and proved that asymptotic consensus can be achieved if the union of the directed graph had a spanning tree frequently enough. Sun et al. [20] investigated the convergence and convergence speed for the second-order and the high-order discrete-time Multiagent systems with random networks and arbitrary weights. Zhang and Tian [21] investigated the mean-square consensus problem for continuous time second-order MASs disturbed by noise, variable delays, and occasional packet dropout and discussed the necessary and sufficient condition for mean-square robust consensusability. And in [22], Zhang and Tian further studied the consensus seeking problem for linear MASs, where each agent communicates via a weighted random lossy network and derived the maximum allowable loss probability.

Motivated by the above analysis, this paper is concerned with consensus problem of general linear discrete-time MASs with random packet dropout that happens during the information exchange between agents, which, to the best of our knowledge, has not been fully investigated until now. The contribution of this paper lies in the following: first, a distributed consensus protocol with weighted graph is proposed to address the packet dropout phenomenon; second, through introducing a new disagreement vector, the dynamic average consensus problem is studied based on the established new framework; third, the necessary and sufficient condition for MASs to reach mean-square consensus is derived in terms of stability of an array of low-dimensional matrices, which is easier to be used in reality; moreover, mean-square consensusable conditions with regard to network topology and agent dynamic structure are also provided.

The rest of this paper is organized as follows. In Section 2 , modeling construction of Multiagent systems with packet dropout is proposed, and some useful lemmas are introduced. In Section 3, a new framework is derived to address the consensus problem of MASs. The consensusable conditions with regard to network topology and agent dynamic structure are presented in Section 4. In Section 5, an illustrative example is provided to demonstrate the effectiveness of our results. Concluding remarks are drawn in Section 6.

Notation. $\mathbb{R}^{n}$ and $\mathbb{R}^{m \times n}$ denote the $n$-dimensional Euclidean space and the set of $m \times n$ real matrix, respectively, and $I_{N}$ represents an $N$-dimensional identity matrix. $\operatorname{diag}\{\cdot\}$ refers to a diagonal matrix, or a block diagonal matrix. For matrices $A$ and $B, A>B$ implies that $A$ and $B$ are symmetric matrices and that $A-B$ is positive definite. The superscript $T$ and -1 mean transpose and inverse of a real matrix separately, and symbol * in a symmetric matrix implies a block that can be induced by symmetry. $\lambda_{\min }(\cdot), \lambda_{\max }(\cdot)$ and $\rho(\cdot)$, denote, respectively, the smallest eigenvalue, the largest eigenvalue and the spectral radius of a matrix. Prob $\{\cdot\}$ stands for the occurrence probability of a stochastic event and $\operatorname{Prob}\{\cdot \mid \cdot\}$ the conditional probability. $E(\cdot)$ is the mathematical expectation operator. $\|\cdot\|$ and $\|\cdot\|_{1}$ stand the 2-norm and 1-norm respectively.

\section{Problem Formulation and Preliminaries}

Consider a system of $N$ agents with identical general linear dynamics. The dynamic equation of agent $i$ is described by

$$
x_{i}(k+1)=A x_{i}(k)+B u_{i}(k), \quad i=1,2, \ldots, N,
$$

where $x_{i}=\left[x_{i 1}, x_{i 2}, \ldots, x_{i n}\right]^{T} \in R^{n}$ is the state variable, $u_{i} \in$ $\mathbb{R}^{m}$ is the control input, and $A \in \mathbb{R}^{n \times n}$ and $B \in \mathbb{R}^{n \times m}$ are known constant matrices.

Suppose that there is a predesigned undirected fixed topology $G$ which guarantees the effective communication between distinct nodes. This assumption is quite reasonable, since it is impossible to reach consensus under an unconnected topology. Denote $N_{i}$ as the neighbor set of the node $i$ and $A_{G}=\left[a_{i j}\right] \in R^{N \times N}$ as the weighted adjacency matrix of undirected graph $G$ with $a_{i i}=0$, and for any $i$ belongs to set $N_{i}, a_{i j}=a_{j i}>0$, otherwise, $a_{i j}=a_{j i}=0$.

Under ideal communication environment, the commonly used linear distributed consensus protocol in MASs is as follows:

$$
u_{i}(k)=K \sum_{j \in N_{i}} a_{i j}\left(x_{j}(k)-x_{i}(k)\right), \quad i=1,2, \ldots, N,
$$

where $K \in \mathbb{R}^{m \times n}$ is the gain matrix.

As mentioned in Section 1, packet dropout is inevitable in practical environment. In this paper, Bernoulli distributed white sequence $\left\{\theta_{i j}(k)\right\}$ is introduced to model the unreliability of the link between agent $i$ and agent $j$. At any given time instant $k$, for any $j \in N_{i}, \theta_{i j}(k)$ takes value in the set $\{0,1\}$. Suppose that for any $i, j \in\{1, \ldots, N\}, \theta_{i j}(k)$ is mutually independent; that is, the packet dropout probability between each pair of agents is independent. When the packet is transmitted successfully between agent $i$ and agent $j, \theta_{i j}(k)=$ 1 ; when packet dropout takes place between agent $i$ and agent $j, \theta_{i j}(k)=0$, which can be described as

$$
\theta_{i j}(k)= \begin{cases}0, & \text { with probability } p_{i j}, \\ 1, & \text { with probability } 1-p_{i j},\end{cases}
$$


where $p_{i j}$ is the occurrence probability of packet dropout between $i$ and $j$, and for any $j \notin N_{i}, \theta_{i j}(k) \equiv 0, p_{i j} \equiv 1$.

Besides, due to the occurrence of packet dropout, the topology of MAS (1) is no longer fixed. Denote $\left|N_{i}\right|$ as the number of agents in set $N_{i}, S$ as the total number of the possible topologies. It is easy to see that $S=2^{\sum_{i=1}^{N}\left|N_{i}\right| / 2}$; then the topology of the MAS is changing among the set $\left\{G_{1}, \ldots, G_{S}\right\}$. Suppose that at certain time instant $k, \theta_{i j}(k)=$ $\theta_{i j}^{m}$ and the network topology is $G_{m}$, then the occurrence probability of graph $G_{m}, m=1, \ldots, S$, is

$$
\pi_{m}=\prod_{i=1, i<j}\left(\theta_{i j}^{m}\left(1-p_{i j}\right)+\left(1-\theta_{i j}^{m}\right) p_{i j}\right) .
$$

Based upon the above analysis, the following distributed consensus protocol is employed in this paper:

$$
u_{i}(k)=K \sum_{j \in N_{i}} \theta_{i j}(k) a_{i j}\left(x_{j}(k)-x_{i}(k)\right), \quad i=1, \ldots, N,
$$

where $K$ is the state feedback gain matrix to be designed.

Substituting (5) into (1), the dynamic equation of agent $i$ can be rewritten as

$$
x_{i}(k+1)=A x_{i}(k)+B K \sum_{j \in N_{i}} \theta_{i j}(k) a_{i j}\left(x_{j}(k)-x_{i}(k)\right) .
$$

Then, the states of MAS (1) evolve according to the following linear system:

$$
x(k+1)=\left(I_{N} \otimes A-L_{\sigma(k)} \otimes(B K)\right) x(k),
$$

where $x=\left[x_{1}^{T}, \ldots, x_{N}^{T}\right]^{T}, \sigma(\cdot): Z^{+} \rightarrow\{1,2, \ldots, S\}$ is a stochastic process driven by an i.i.d. process and $L_{\sigma(k)} \in\left\{L_{m}\right\}$, where $L_{m}=\left[l_{i j}^{m}\right]_{N \times N}, m=1, \ldots, S$, is the Laplacian matrix associated with $G_{m}$, with

$$
l_{i j}^{m}= \begin{cases}\sum_{j=1}^{N} \theta_{i j}^{m} a_{i j}, & i=j, \\ -\theta_{i j}^{m} a_{i j}, & i \neq j .\end{cases}
$$

Obviously, the Laplacian matrix $L_{m}$ is a symmetric matrix with zero row sum that is, $\forall i \neq j, l_{i j}=l_{j i}$, and $\sum_{j=1}^{N} l_{i j}^{m}=0$.

Before presenting our main results, the concepts of meansquare stability and mean-square consensus are introduced first, and along with them are some useful lemmas which play significant roles in the derivation of our results.

Definition 1 (see [23]). A closed-loop system is said to be mean-square stable, if for any $\varepsilon>0$, there exists $\delta(\varepsilon)>0$ such that for any $k>0, E\left(\|x(k)\|^{2}\right)<\varepsilon$ holds when $E\left(\|x(0)\|^{2}\right)<$ $\delta(\varepsilon)$. In addition, if $\lim _{k \rightarrow \infty} E\left(\|x(k)\|^{2}\right)=0$ for any initial condition, then the closed-loop system is said to be globally mean-square asymptotically stable.

Definition 2. Multiagent system (1) with protocol (5) is said to reach consensus in mean-square sense, if for any initial distribution and initial states, $\lim _{k \rightarrow \infty} E\left(\left\|x_{i}(k)-x_{j}(k)\right\|^{2}\right)=$ 0 holds for all $i, j \in\{1, \ldots, N\}$. Furthermore, if there exists a proper state feedback gain matrix $K$ such that the Multiagent system can achieve mean-square consensus, then we say that system (1) is mean-square consensusable under protocol (5).

Lemma 3 (see [21]). For any vector $z(k) \in \mathbb{R}^{n}$, denote $\bar{z}(k):=$ $E(z(k) \otimes z(k))$, then there is

$$
E\left(\|z(k)\|^{2}\right) \leq\|\bar{z}(k)\|_{1} \leq n E\left(\|z(k)\|^{2}\right) .
$$

Lemma 4 (see [6]). For an undirected graph with $n$ vertices, the Laplacian is a positive-semidefinite symmetric matrix, and the spectrum of a graph Laplacian is on the form

$$
0=\lambda_{1}(L) \leq \lambda_{2}(L) \leq \cdots \leq \lambda_{n-1}(L) \leq \lambda_{n}(L),
$$

with $\lambda_{2}(L)>0$ if and only if the graph that corresponds to $L$ is connected.

Lemma 5. $\sum_{m=1}^{S} \pi_{m} L_{m}$ has only one zero eigenvalue, if and only if the union graph of the topology set $\left\{G_{1}, \ldots, G_{S}\right\}$ is connected.

Proof. Since $\sum_{m=1}^{S} \pi_{m} L_{m}$ can be treated as a Laplacian matrix of a graph which has the same edges with the union graph of the topology set $\left\{G_{1}, \ldots, G_{S}\right\}$, then together with Lemma 4 , it can be obtained that Lemma 5 holds.

Lemma 6 (see [24]). For any $A \in \mathbb{R}^{n \times n}$, there exist orthogonal matrix $U$ and an upper triangular matrix $V$, such that

$$
U^{T} A U=V=\left[\begin{array}{cccc}
\mu_{1} & v_{12} & \cdots & v_{1 n} \\
& \mu_{2} & \cdots & v_{2 n} \\
& & \ddots & \vdots \\
& & & \mu_{n}
\end{array}\right],
$$

where $\mu_{i}, i=1, \ldots, n$, are the eigenvalues of matrix $A$.

\section{Necessary and Sufficient Conditions for Mean-Square Consensus}

Introduce the following variable:

$$
\delta_{i}(k)=x_{i}(k)-\frac{1}{N} \sum_{j=1}^{N} x_{j}(k) .
$$

Rewrite (12) in a compact form, which is

$$
\delta(k)=\left(C \otimes I_{n}\right) x(k),
$$

where $C=I_{N}-(1 / N) \overrightarrow{1} \overrightarrow{1}^{T}$ and $\delta=\left[\delta_{1}^{T}, \ldots, \delta_{N}^{T}\right]^{T} \in R^{N n}$ is the so-called disagreement vector.

It is easy to verify that $\delta$ evolves according to the following disagreement dynamics:

$$
\delta(k+1)=\left(I_{N} \otimes A-L_{\sigma(k)} \otimes(B K)\right) \delta(k),
$$

where $\delta(k)$ is defined in (7). 
Since $E\left(\|\delta(k)\|^{2}\right)=E\left(x^{T}(k)\left(Q \otimes I_{n}\right) x(k)\right)$, where $Q=$ $C^{T} C=I_{N}-(1 / N) \overrightarrow{1} \overrightarrow{1}^{T}$, it is easy to verify that

$$
E\left(\|\delta(k)\|^{2}\right)=\frac{1}{2 N} \sum_{i \neq j} E\left(\left\|x_{j}(k)-x_{i}(k)\right\|^{2}\right) .
$$

Equation (15) implies that the mean-square consensus problem can be solved if and only if $\lim _{k \rightarrow \infty} E\left(\|\delta(k)\|^{2}\right)=0$. From Definitions 1 and 2, it is easy to see that the consensus problem of MAS (1) is converted to the mean square stability analysis of system (14).

Remark 7. If the system matrix $A$ is Schur stable, which means all eigenvalues of $A$ are located in the unit circle, MAS (1) would definitely reach consensus without the design of consensus protocol, since under this condition, for any $i \in\{1, \ldots, N\}, x_{i}$ will converge to zero, and $\lim _{k \rightarrow \infty} E\left(\left\|x_{i}(k)-x_{j}(k)\right\|^{2}\right)=0$ holds for sure. In view of this, we assume that not all eigenvalues of $A$ are located in the unit circle.

Theorem 8. For any initial distribution and initial states, Multiagent system (1) with the Bernoulli packet dropout between agents can achieve mean-square consensus under protocol (1), if and only if

$$
\rho\left(A-\widehat{\lambda}_{i} B K\right)<1
$$

where $\hat{\lambda}_{i}, i=2, \ldots, N$, are the eigenvalues of matrix $\sum_{m=1}^{S} \pi_{m} L_{m}$ except one zero eigenvalue.

Proof. Since for each $\sigma(k)=m, m=1, \ldots, S, L_{\sigma(k)}$ is a symmetric matrix with zero row sum, and $w^{T}=(1 / N) \overrightarrow{1}^{T}$ and $\overrightarrow{1}$ are, respectively, the left and right eigenvectors of $L_{\sigma(k)}$ associated with zero eigenvalue, we can construct an invertible matrix $T=\left[\begin{array}{ll}\overrightarrow{1} & T_{1}\end{array}\right]$ with $T^{-1}=\left[\begin{array}{ll}w & T_{2}^{T}\end{array}\right]^{T}$, such that

$$
T^{-1} L_{\sigma(k)} T=\bar{L}_{\sigma(k)}=\left[\begin{array}{cc}
0 & 0 \\
0 & \widetilde{L}_{\sigma(k)}
\end{array}\right],
$$

where $T \in \mathbb{R}^{N \times N}, T_{1} \in \mathbb{R}^{N \times(N-1)}, T_{2} \in \mathbb{R}^{(N-1) \times N}$, and $\tilde{L}_{\sigma(k)}=T_{2} L_{\sigma(k)} T_{1} \in R^{(N-1) \times(N-1)}$.

Introduce the following variable: $\varphi(k)=\left(T^{-1} \otimes I_{n}\right) \delta(k)$, where $\varphi=\left[\varphi_{1}^{T}, \ldots, \varphi_{N}^{T}\right]^{T} \in R^{N n}$; then (14) can be denoted in terms of $\varphi$ as

$$
\varphi(k+1)=\left(I_{N} \otimes A-\bar{L}_{\sigma(k)} \otimes(B K)\right) \varphi(k) .
$$

Note that $\varphi_{1}=\left(w^{T} \otimes I_{n}\right) \delta \equiv 0$, which implies that state $\varphi_{1}$ is always stable; thus, divide $\varphi(k)$ into two parts; that is, $\varphi(k)=\left[\varphi_{1}^{T}(k), \hat{\varphi}^{T}(k)\right]^{T}$, with $\widehat{\varphi}=\left[\varphi_{2}^{T}, \ldots, \varphi_{N}^{T}\right]^{T}$, it can be derived from (17) and (18) that

$$
\widehat{\varphi}(k+1)=\widetilde{A}_{\sigma(k)} \widehat{\varphi}(k),
$$

where $\widetilde{A}_{\sigma(k)}=I_{N-1} \otimes A-\widetilde{L}_{\sigma(k)} \otimes(B K)$.
Similar to [21], let $\xi(k)=E(\widetilde{\varphi}(k) \otimes \widetilde{\varphi}(k))$; then $\xi(k)$ evolves according to

$$
\xi(k+1)=\sum_{m=1}^{S} \pi_{m}\left(\widetilde{A}_{m} \otimes \widetilde{A}_{m}\right) \xi(k) .
$$

From Lemma 3, system (14) is mean-square stable if and only if system (20) is stable. Then, from the discrete-time system stability theory, it can be concluded that the necessary and sufficient condition for mean-square stability of (14) is $\rho\left(\sum_{m=1}^{S} \pi_{m}\left(\widetilde{A}_{m} \otimes \widetilde{A}_{m}\right)\right)<1$.

Denote $\widetilde{A}_{m}=\Phi-\Psi_{m} K$ with $\Phi=I_{N-1} \otimes A, \Psi_{m}=\widetilde{L}_{m} \otimes B$, $K=I_{N-1} \otimes K$, and $K=\varepsilon K^{\prime}$; then there is $\sum_{m=1}^{S} \pi_{m}\left(\widetilde{A}_{m} \otimes\right.$ $\left.\widetilde{A}_{m}\right)=\Phi \otimes \Phi-\varepsilon\left(\sum_{m=1}^{S} \pi_{m} \Phi \otimes\left(\Psi_{m} K^{\prime}\right)+\sum_{m=1}^{S} \pi_{m}\left(\Psi_{m} K^{\prime}\right) \otimes \Phi\right)$ $+\varepsilon^{2} \sum_{m=1}^{S} \pi_{m}\left(\Psi_{m} K^{\prime}\right) \otimes\left(\Psi_{m} K^{\prime}\right)$. The above equation can be treated as a perturbation of $\Phi \otimes \Phi$ by two terms depending on $\varepsilon$. For small enough $\varepsilon$, the above formula is equal to $\sum_{m=1}^{S} \pi_{m}\left(\widetilde{A}_{m} \otimes \widetilde{A}_{m}\right)=\Phi \otimes \Phi-\varepsilon\left(\sum_{m=1}^{S} \pi_{m} \Phi \otimes\right.$ $\left.\left(\Psi_{m} K^{\prime}\right)+\sum_{m=1}^{S} \pi_{m}\left(\Psi_{m} K^{\prime}\right) \otimes \Phi\right)$. Meanwhile, $\left(\sum_{m=1}^{S} \pi_{m} \widetilde{A}_{m}\right) \otimes$ $\left(\sum_{m=1}^{S} \pi_{m} \widetilde{A}_{m}\right)=\Phi \otimes \Phi-\varepsilon\left(\sum_{m=1}^{S} \pi_{m} \Phi \otimes\left(\Psi_{m} K^{\prime}\right)+\right.$ $\left.\sum_{m=1}^{S} \pi_{m}\left(\Psi_{m} K^{\prime}\right) \otimes \Phi\right)+\varepsilon^{2} \sum_{m=1}^{S}\left(\pi_{m} \Psi_{m} K^{\prime}\right) \otimes\left(\pi_{m} \Psi_{m} K^{\prime}\right)$. Likewise, by applying perturbation argument, for a small enough $\varepsilon, \rho\left(\sum_{m=1}^{S} \pi_{m}\left(\widetilde{A}_{m} \otimes \widetilde{A}_{m}\right)\right)<1$ is equivalent to

$$
\rho\left(\left(\sum_{m=1}^{S} \pi_{m} \widetilde{A}_{m}\right) \otimes\left(\sum_{m=1}^{S} \pi_{m} \widetilde{A}_{m}\right)\right)<1 .
$$

Next, it will be proved that (21) holds if and only if (16) is satisfied.

It can be seen from (18) that $\sum_{m=1}^{S} \pi_{m} \widetilde{L}_{m}$ has the same eigenvalue with $\sum_{m=1}^{S} \pi_{m} \bar{L}_{m}$ and $\sum_{m=1}^{S} \pi_{m} L_{m}$ except one zero eigenvalue. Besides, based on Lemma 6 , there exists an orthogonal matrix $U \in R^{(N-1) \times(N-1)}$ such that

$$
U^{T}\left(\sum_{m=1}^{S} \pi_{m} \widetilde{L}_{m}\right) U=\left[\begin{array}{cccc}
\hat{\lambda}_{2} & * & \cdots & * \\
& \hat{\lambda}_{3} & \cdots & * \\
& & & \vdots \\
& & & \hat{\lambda}_{N}
\end{array}\right],
$$

where $\hat{\lambda}_{i}, i=2, \ldots, N$, are the eigenvalues of matrix $\sum_{m=1}^{S} \pi_{m} L_{m}$ except one zero eigenvalue. It is easy to verify that $\sum_{m=1}^{S} \pi_{m} \widetilde{A}_{m}=I_{N-1} \otimes A-\sum_{m=1}^{S} \pi_{m} \widetilde{L}_{m} \otimes(B K)$ is similar to

$$
\left[\begin{array}{cccc}
A-\hat{\lambda}_{2} B K & * & \cdots & * \\
& A-\widehat{\lambda}_{3} B K & \cdots & * \\
& & & \ddots \\
& & & A-\widehat{\lambda}_{N} B K
\end{array}\right] \text {. }
$$

Based on the matrix theory, matrix $\sum_{m=1}^{S} \pi_{m} \widetilde{A}_{m}$ has the same eigenvalue with matrix (23), obviously $\rho\left(\sum_{m=1}^{S} \pi_{m} \widetilde{A}_{m}\right)<1$, if and only if (16) is satisfied. Then, together with the properties of the Kronecker product, it is safe to say that (21) holds if and only if (16) is satisfied, which completes the proof. 
Remark 9. The contribution of Theorem 8 lies in the fact that it converts the consensus problem of MAS (1) into a set of matrices with the same dimensions as a single agent, which reduces the computational complexity greatly. Besides, while in most of the existing results each agent is guaranteed to achieve consensus with agent 1 , see $[7,11,21]$ for example, or to achieve average consensus, see [5], for example, in this paper a new disagreement vector is introduced to solve the consensus problem so that each agent will reach consensus on the average states of all agents, that is, dynamic average consensus.

\section{Consensusability Analysis}

Theorem 8 presents the numerical solution to consensus problem, which also implies the design method of consensus protocol. However, in practice, we are equally interested in that under what conditions MAS (1) has the ability to reach consensus and inequality (16) has a solution at the same time, that is, what are the consensusable conditions? In this section, we focus on addressing this problem.

Theorem 10. Multiagent system (1) with the Bernoulli packet dropout between agents can achieve mean-square consensus under protocol (5), if and only if the union graph of the topology set $\left\{G_{1}, \ldots, G_{S}\right\}$ is connected, and $(A, B)$ is stabilizable.

Proof. Necessity: define $E\left(L_{\sigma(k)}\right)=\sum_{m=1}^{S} \pi_{m} L_{m}$ as the expected Laplacian matrix and its corresponding graph as the expected graph. Since $E\left(L_{\sigma(k)}\right)$ is a symmetric matrix, there exists an invertible matrix $\widehat{T}=\left[\begin{array}{ll}\overrightarrow{1} & \widehat{F}\end{array}\right]$ with $\widehat{T}^{-1}=\left[\begin{array}{ll}w & \widehat{E}^{T}\end{array}\right]^{T}$, such that

$$
\widehat{T}^{-1} E\left(L_{\sigma(k)}\right) \widehat{T}=\Lambda=\left[\begin{array}{ll}
0 & 0 \\
0 & \widehat{\Delta}
\end{array}\right],
$$

where $\widehat{T} \in \mathbb{R}^{N \times N}, \widehat{F} \in \mathbb{R}^{N \times(N-1)}, \widehat{E} \in \mathbb{R}^{(N-1) \times N}, w^{T}$, which is defined in (18), is also the left eigenvalue of $E\left(L_{\sigma(k)}\right)$ associated with zero eigenvalue, and $\widehat{\Delta} \in R^{(N-1) \times(N-1)}$ is a diagonal matrix with its diagonal elements being $\hat{\lambda}_{i}, i=$ $2, \ldots, N$.

Denote $\phi(k)=E(\delta(k))$; then $\phi(k)$ evolves according to

$$
\phi(k+1)=\left(I_{N} \otimes A-E\left(L_{\sigma(k)}\right) \otimes(B K)\right) \phi(k),
$$

where $\phi=\left[\phi_{1}^{T}, \ldots, \phi_{N}^{T}\right]^{T} \in R^{N n}$.

Similar to the proof in Theorem 8 , introduce the following variable transformation: $\zeta(k)=\left(\widetilde{T}^{-1} \otimes I_{n}\right) \phi(k)$; then the dynamic equation of $\zeta(k)$ can be written as

$$
\zeta(k+1)=\left(I_{N} \otimes A-\Lambda \otimes(B K)\right) \zeta(k)
$$

where $\zeta=\left[\zeta_{1}^{T}, \ldots, \zeta_{N}^{T}\right]^{T} \in R^{N n}$.
Notice that $\zeta_{1}(k)=\left(\widetilde{w}^{T} \otimes I_{N}\right) E(\delta(k)) \equiv 0$, divide $\zeta(k)=\left(\widetilde{w}^{T} \otimes I_{N}\right) E(\delta(k)) \equiv 0$ into two parts, that is, $\zeta(k)=$ $\left[\zeta_{1}^{T}(k), \widetilde{\zeta}^{T}(k)\right]^{T}$, from (19) and (21), it can be derived that

$$
\widetilde{\zeta}(k+1)=\left[\begin{array}{cccc}
A-\hat{\lambda}_{2} B K & 0 & \cdots & 0 \\
& A-\widehat{\lambda}_{3} B K & \cdots & 0 \\
& & \ddots & \vdots \\
& & & A-\hat{\lambda}_{N} B K
\end{array}\right] \widetilde{\zeta}(k) .
$$

If the union graph of the topology set $\left\{G_{1}, \ldots, G_{S}\right\}$ is not connected, the expected graph is definitely not connected. It can be obtained from Lemma 3 that $0=\widehat{\lambda}_{2} \leq \widehat{\lambda}_{3} \leq$ $\cdots \leq \widehat{\lambda}_{N}$. Due to the fact that not all eigenvalues of $A$ are located in the unit circle, there exists an initial state such that $\lim _{k \rightarrow \infty} \zeta(k) \neq 0$ and $\lim _{k \rightarrow \infty} E(\delta(k)) \neq 0$ thus; system (14) is not mean-square stable since $E\left(\|\delta(k)\|^{2}\right) \geq\|E(\delta(k))\|^{2}$, and consequently, Multiagent system (1) cannot achieve meansquare consensus.

Besides, if $(A, B)$ is unstabilizable, it is impossible to guarantee that $\rho\left(A-\widehat{\lambda}_{i} B K\right)<1, i=2, \ldots, N$, even if the topology condition is satisfied, and system (27) is still not guaranteed to be stable.

Sufficiency: It can be derived from Lemma 5 that when the union of the graphs in the topology set is connected, all eigenvalues of $\sum_{m=1}^{S} \pi_{m} \widetilde{L}_{m}$ are positive; that is, $\hat{\lambda}_{i}>0$, $i=2, \ldots, N$. Therefore, when the union graph of the topology set $\left\{G_{1}, \ldots, G_{S}\right\}$ is connected and $(A, B)$ is stablizable, there exists a proper gain matrix $K$ such that $\rho\left(A-\hat{\lambda}_{i} B K\right)<1$, $i=2, \ldots, N$. The proof is completed.

Remark 11. In [21], for two-order continuous-time MASs with $A=\left[\begin{array}{ll}0 & 1 \\ 0 & 0\end{array}\right], B=\left[\begin{array}{l}0 \\ 1\end{array}\right]$, which implies that $(A, B)$ is stablizable, Zhang and Tian prove that the MAS can reach mean-square consensus if and only if the union graph is connected, while in this paper, it is verified theoretically that the stabilizability of $(A, B)$ is essential for achieving consensus.

\section{Numerical Simulation}

Consider an MAS consisted of four agents, with its prior fixed weighted communication topology being $N_{1}=\{2\}, N_{2}=$ $\{1,3\}, N_{3}=\{2,4\}, N_{4}=\{3\}, a_{12}=2, a_{23}=2, a_{34}=3$; then there are eight graphs in the topology set $\left\{G_{1}, \ldots, G_{8}\right\}$. Assume that the packet dropout probability between each pair of agents is 0.1 .

Each agent is described by a three-order difference equation, with

$$
A=\left[\begin{array}{ccc}
0.6 & 0 & 0.2 \\
0.3 & 0.3 & 0.4 \\
0 & 0 & 1.2
\end{array}\right], \quad B=\left[\begin{array}{l}
0 \\
0 \\
1
\end{array}\right]
$$

Obviously, each agent is unstable since the eigenvalues of $A$ are $0.6,0.3$, and 1.2 , and $(A, B)$ is stablizable. By applying Theorem 8 , it can be obtained that 


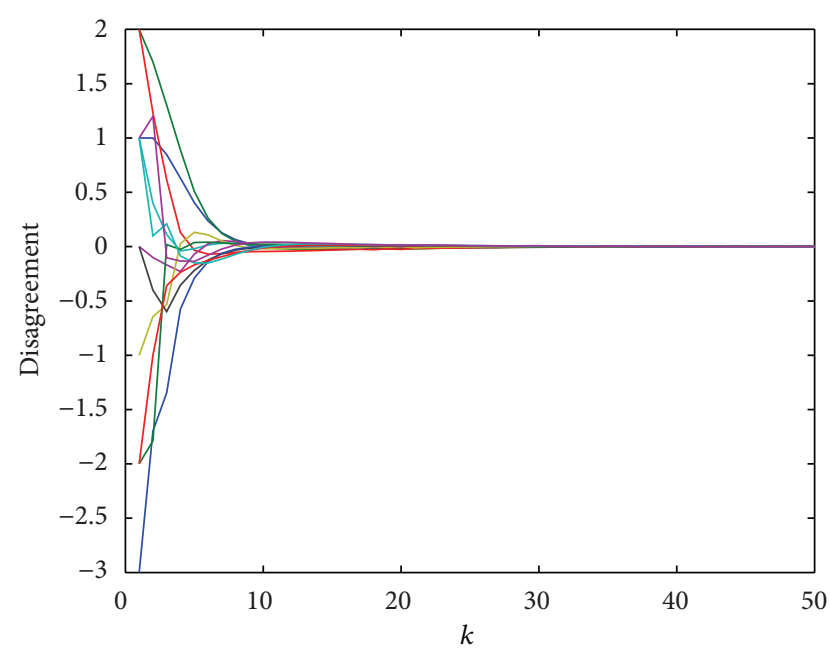

FIGURE 1: The disagreement values between agents under consensus protocol (5).

$K=\left[\begin{array}{ll}0.1111-0.0000 & 0.1944\end{array}\right]$, such that $\rho\left(A-\hat{\lambda}_{i} B K\right)=$ $0.8910<1$. Suppose the initial conditions are $x_{10}=$ $\left[\begin{array}{lll}2 & 3 & 2\end{array}\right]^{T}, x_{20}=\left[\begin{array}{ccc}2 & 1 & -1\end{array}\right]^{T}, x_{30}=\left[\begin{array}{lll}1 & -2 & -3\end{array}\right]^{T}$, and $x_{40}=$ $\left[\begin{array}{lll}-1 & 2 & -2\end{array}\right]^{T}$; the simulation results are shown in Figure 1.

\section{Conclusion}

We have studied the consensus problem of general linear discrete-time Multiagent systems (MASs) with random packet dropout that happens during information exchange between agents. The packet dropout phenomenon is characterized as being a Bernoulli random process. A distributed consensus protocol with weighted graph is proposed to address the packet dropout phenomenon. By constructing a new disagreement vector, a new framework is established to solve the consensus problem. Then, through introducing a common linear transformation for the switching system, together with the control theory, the perturbation argument, and the matrix theory, the necessary and sufficient condition for MASs to reach mean-square consensus is derived in terms of stability of an array of low-dimensional matrices. Moreover, mean-square consensusable conditions with regard to network topology and agent dynamic structure are also provided.

It is worth pointing out that this paper is only a first step; the consensus problem of linear discrete time MASs, which may be affected by many other factors including time-delay, and noise, is not fully investigated, and this is of our research interest in the future.

\section{Acknowledgments}

This work is supported by the National Natural Science Foundation of China under Grants 61074124 and 61374171, the Project-Sponsored by SRF for ROCS, SEM, the Fundamental Research Funds for the Central Universities, HUST, under
Grant 2012TS020, and the Graduate Fund of Innovation and Entrepreneurship, Hust, under Grant HF-11-18-2013.

\section{References}

[1] S. Kar and J. M. F. Moura, "Distributed consensus algorithms in sensor networks: quantized data and random link failures," IEEE Transactions on Signal Processing, vol. 58, no. 3, pp. 13831400, 2010.

[2] N. A. Lynch, Distributed Algorithms, Morgan Kaufmann, San Francisco, Calif, USA, 1997.

[3] H. G. Tanner, A. Jadbabaie, and G. J. Pappas, "Flocking in fixed and switching networks," IEEE Transactions on Automatic Control, vol. 52, no. 5, pp. 863-868, 2007.

[4] R. S. Dong and Z. Y. Geng, "Consensus based formation control laws for systems on Lie groups," Systems \& Control Letters, vol. 62, no. 2, pp. 104-111, 2013.

[5] R. Olfati-Saber and R. M. Murray, "Consensus problems in networks of agents with switching topology and time-delays," IEEE Transactions on Automatic Control, vol. 49, no. 9, pp. 15201533, 2004.

[6] Y. Hatano and M. Mesbahi, "Agreement over random networks," IEEE Transactions on Automatic Control, vol. 50, no. 11, pp. 1867-1872, 2005.

[7] Y. Zhang and Y.-P. Tian, "Consentability and protocol design of multi-agent systems with stochastic switching topology," Automatica, vol. 45, no. 5, pp. 1195-1201, 2009.

[8] F. Sun, J. Chen, Z.-H. Guan, L. Ding, and T. Li, "Leaderfollowing finite-time consensus for multi-agent systems with jointly-reachable leader," Nonlinear Analysis: Real World Applications, vol. 13, no. 5, pp. 2271-2284, 2012.

[9] P. Lin, Z. Li, Y. Jia, and M. Sun, "High-order multi-agent consensus with dynamically changing topologies and timedelays," IET Control Theory \& Applications, vol. 5, no. 8, pp. 976981, 2011.

[10] Z. K. Li, Z. S. Duan, G. R. Chen, and L. Hang, "Consensus of multi-agent systems and synchronization of complex networks: a unified viewpoint," IEEE Transactions on Circuits and Systems, vol. 57, no. 1, pp. 213-224, 2010.

[11] J. Xu, L. H. Xie, T. Li, and K. Y. Lum, "Consensus of multiagent systems with general linear dynamics via dynamic output feedback control," IET Control Theory Applications, vol. 7, no. 1, pp. 108-115, 2013.

[12] X. L. Xu, S. Y. Cheng, W. Huang, and L. X. Gao, "Leaderfollowing consensus of discrete-time multi-agent systems with observer-based protocols," Neurocomputing, vol. 118, pp. 334$341,2013$.

[13] Y. F. Su and J. Huang, "Two consensus problems for discretetime multi-agent systems with switching network topology," Automatica, vol. 48, no. 9, pp. 1988-1997, 2012.

[14] P. Lin and Y. Jia, "Multi-agent consensus with diverse timedelays and jointly-connected topologies," Automatica, vol. 47, no. 4, pp. 848-856, 2011.

[15] Z. H. Wu and H. J. Fang, "Improvement for consensus performance of multi-agent systems based on delayed-state-derivative feedback," Journal of Systems Engineering and Electronics, vol. 23, no. 1, pp. 137-144, 2012.

[16] Z. H. Wu, L. Peng, L. B. Xie, and J. W. Wen, "Stochastic bounded consensus tracking of leader-follower multi-agent systems with measurement noises based on sampled-data with 
small sampling delay," Physica A, vol. 392, no. 4, pp. 918-928, 2013.

[17] W. Zhu, "Consensus of multiagent systems with switching jointly reachable interconnection and time delays," IEEE Transactions on Systems, Man, and Cybernetics A, vol. 42, no. 2, pp. 348-358, 2012.

[18] Y. G. Sun and L. Wang, " $H_{\infty}$ consensus of second-order multiagent systems with asymmetric delays," Systems \& Control Letters, vol. 61, no. 8, pp. 857-862, 2012.

[19] W. Ren and R. W. Beard, "Consensus seeking in multiagent systems under dynamically changing interaction topologies," IEEE Transactions on Automatic Control, vol. 50, no. 5, pp. 655-661, 2005.

[20] F. L. Sun, Z.-H. Guan, X.-S. Zhan, and F.-S. Yuan, "Consensus of second-order and high-order discrete-time multi-agent systems with random networks," Nonlinear Analysis: Real World Applications, vol. 13, no. 5, pp. 1979-1990, 2012.

[21] Y. Zhang and Y.-P. Tian, "Consensus of data-sampled multiagent systems with random communication delay and packet loss," IEEE Transactions on Automatic Control, vol. 55, no. 4, pp. 939-943, 2010.

[22] Y. Zhang and Y.-P. Tian, "Maximum allowable loss probability for consensus of multi-agent systems over random weighted lossy networks," IEEE Transactions on Automatic Control, vol. 57, no. 8, pp. 2127-2132, 2012.

[23] C. Z. Zhang, G. Feng, H. J. Gao, and Y. Zhao, "State feedback controller design of networked control systems with multiple packet dropouts," in Proceedings of the 29th Chinese Control Conference (CCC '10), pp. 5839-5844, July 2010.

[24] F. Z. Zhang, Matrix Theory, Springer, New York, NY, USA, 1999. 


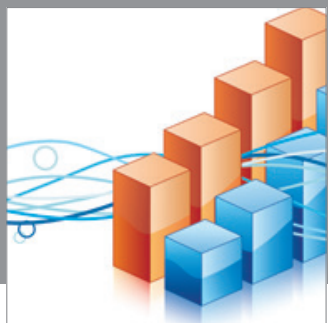

Advances in

Operations Research

mansans

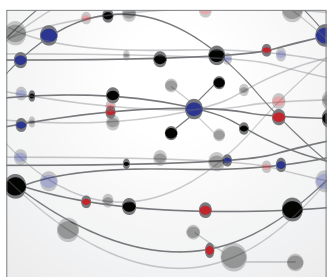

The Scientific World Journal
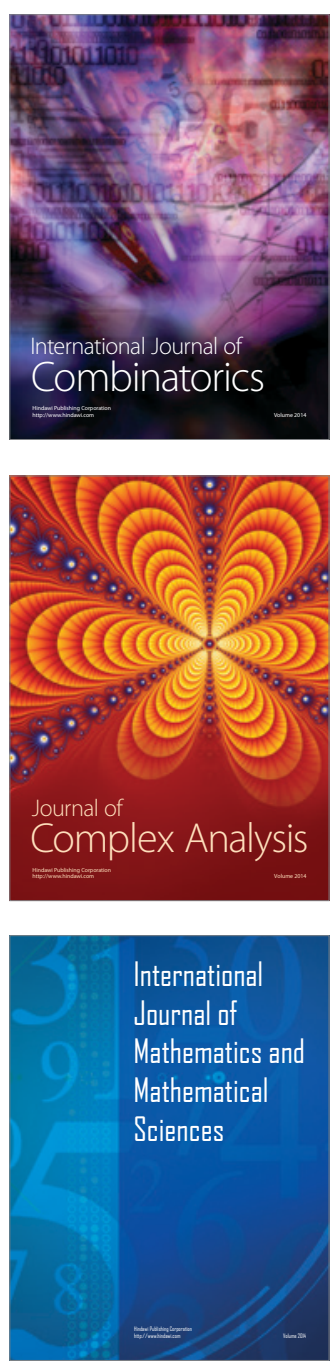
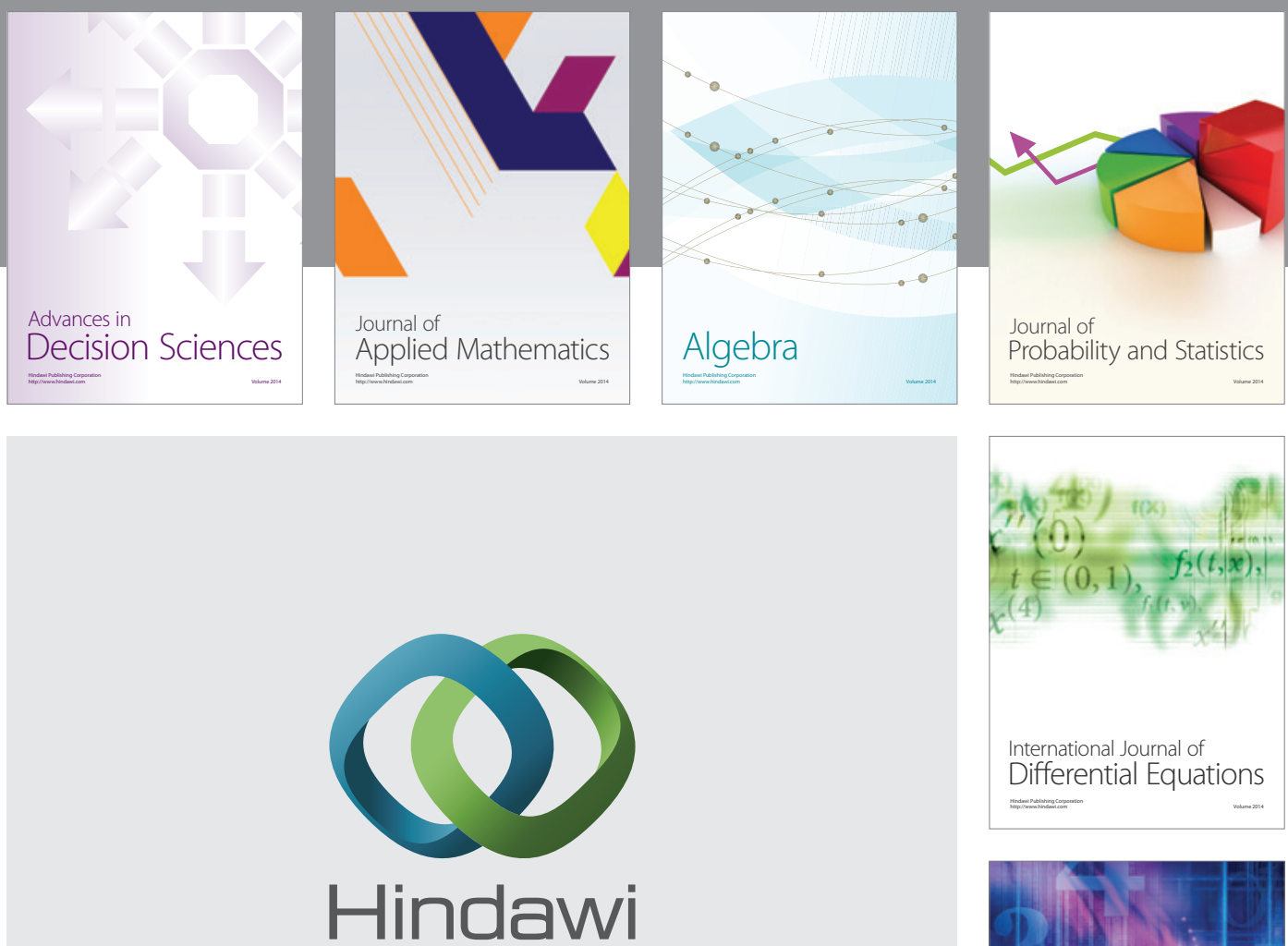

Submit your manuscripts at http://www.hindawi.com
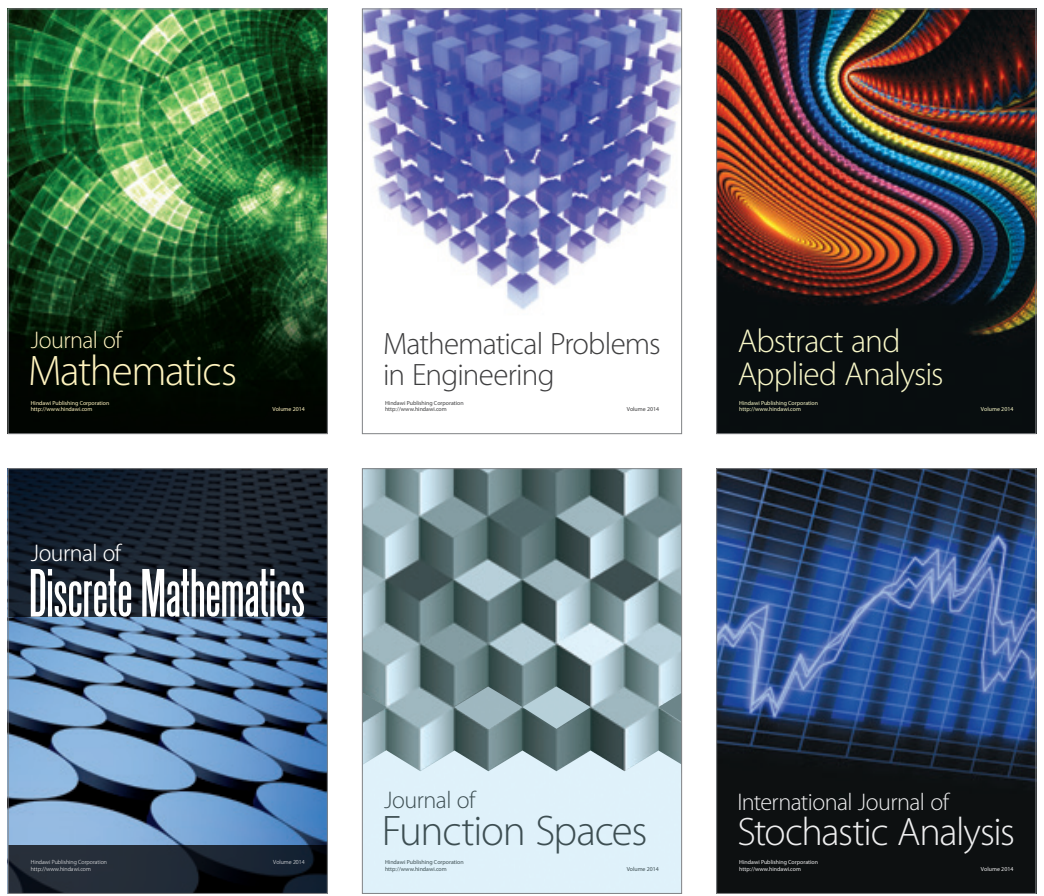

Journal of

Function Spaces

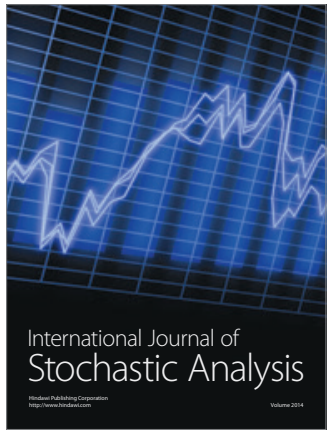

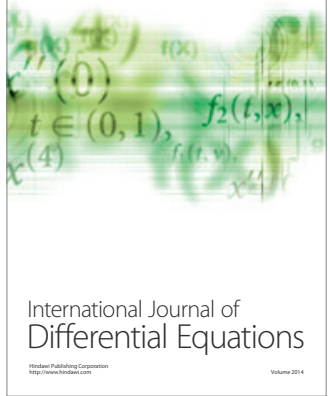
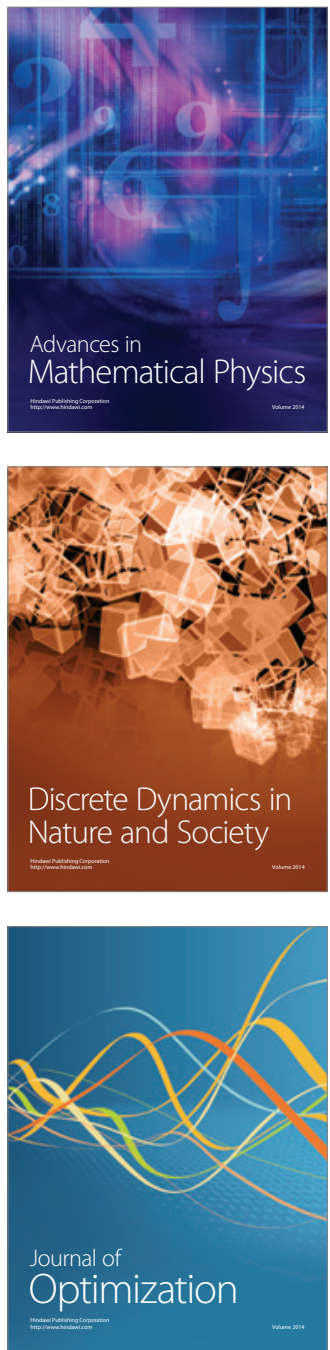